\title{
Diplopia, Proptosis, and Joint Pain: Possibility of Osseous and Orbital Sarcoidosis
}

RITESH KOHLI, MD, Rheumatologist, Marietta Rheumatology Associates, Atlanta, Georgia; CHOKKALINGAM SIVA, MD, MS, Director, Rheumatology Fellowship Program, University of Missouri, Columbia, Missouri, USA. Address correspondence to Dr. R. Kohli, Rheumatologist, Marietta Rheumatology Associates, 855 Emory Point Dr., Apt. 3434, Atlanta, Georgia 30329, USA. E-mail: riteshkohli@ yahoo.com. Ethical board approval was not required in accordance with the policy of the institution. J Rheumatol 2018;45:141-2; doi:10.3899/jrheum.170439

Osseous sarcoidosis is associated with systemic involvement, aggressive disease, and poor prognosis ${ }^{1}$, and is often discovered through radiographs. Orbital sarcoidosis is uncommon, but may present in disseminated sarcoidosis ${ }^{2}$.

A 28-year-old man was referred by an ophthalmologist to the rheumatology clinic with a history of diplopia and visual blurring. He had recurrent right eye pain and eyelid swelling for 1 year that did not respond to topical antiinflammatory agents. He also complained of episodic joint pain and swelling involving hands and feet, dyspnea on exertion, and periodic left lower extremity weakness.

Physical examination revealed right eye proptosis, upper gaze palsy, axillary and supraclavicular lymphadenopathy, and diffuse subcutaneous nodules in the hands.

Investigation revealed elevated angiotensin-converting enzyme level of $103 \mathrm{U} / 1$ (normal range 8-53 U/1). Orbital magnetic resonance imaging (MRI) scan demonstrated infiltrative lesions in right inferior rectus muscle and lacrimal glands (Figure 1). Biopsy of axillary lymph node revealed noncaseating granulomas. Enhancing lesions in vertebral body, posterior elements, and spinal cord were seen in MRI spine. Hand radiographs showed extensive lytic lesions involving the phalanges and pathologic fractures (Figure 2). Diplopia and joint inflammation were resolved with prednisone $60 \mathrm{mg}$ daily and azathioprine $150 \mathrm{mg}$ daily.

Osseous sarcoidosis is associated with systemic involvement, aggressive disease, and poor prognosis ${ }^{1}$. Orbital sarcoidosis is uncommon but may present as an urgent clinical problem in disseminated sarcoidosis ${ }^{2}$. Musculoskeletal manifestations of sarcoidosis include arthralgias, arthritis, and osseous sarcoidosis. Unlike sarcoid arthritis, where inflammation is limited to the joints, in osseous

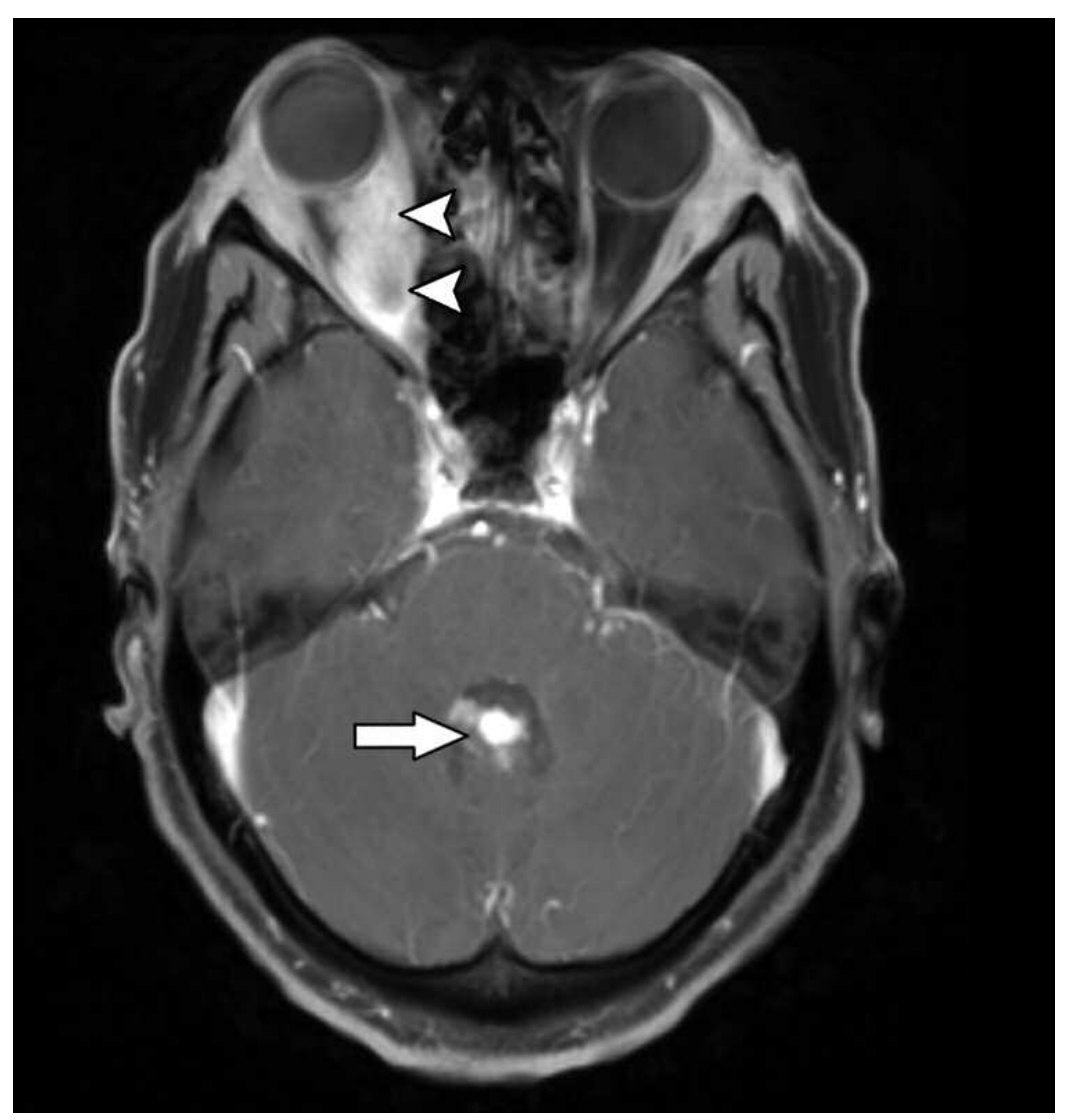

Figure 1. Magnetic resonance imaging of the brain. Axial T1-weighted post-contrast image shows multifocal and nodular enhancement of intraventricular (arrow) and intraorbitary/dural lesions (arrowheads).

Personal non-commercial use only. The Journal of Rheumatology Copyright (C) 2018. All rights reserved. 


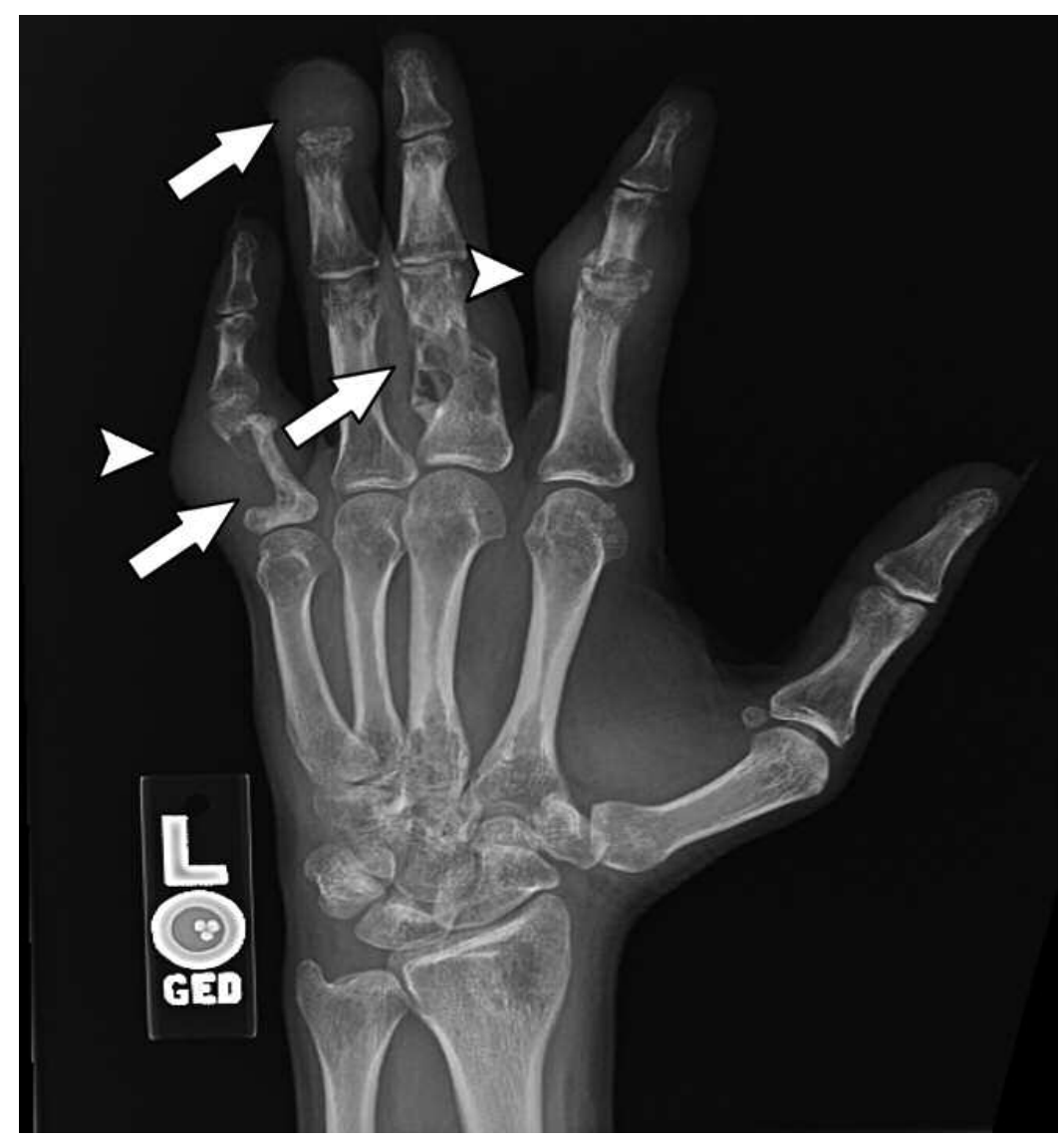

Figure 2. Radiograph of the hand in anteroposterior view shows multifocal aggressive osseous lesions with pathologic fractures (arrows) and soft tissue nodularity (arrowheads).

sarcoidosis the shafts of the bones are involved, often leading to lytic, destructive lesions ${ }^{3}$. Many patients with osseous sarcoidosis may be asymptomatic and the lesions may be discovered incidentally on radiographs.

\section{ACKNOWLEDGMENT}

We acknowledge the significant contribution of Atul Taneja, MD, PhD, Musculoskeletal Radiology Division, Hospital Israelita Albert Einstein, São Paulo, Brazil.

\section{REFERENCES}

1. Chatham W. Rheumatic manifestations of systemic disease: sarcoidosis. Curr Opin Rheumatol 2010;22:85-90.

2. Pasadhika S, Rosenbaum JT. Ocular sarcoidosis. Clin Chest Med 2015;36:669-83.

3. Sparks JA, McSparron JI, Shah N, Aliabadi P, Paulson V, Fanta CH, et al. Osseous sarcoidosis: clinical characteristics, treatment, and outcomes-experience from a large, academic hospital. Semin Arthritis Rheum 2014;44:371-9. 